\title{
New Catalytic Reactions Using Sulfur Dioxide
}

\section{Michael C. Willis}

Department of Chemistry, University of Oxford, Chemistry Research Laboratory, Mansfield Road, Oxford, OX1

3TA, United Kingdom.

michael.willis@chem.ox.ac.uk

ABSTRACT The sulfur dioxide surrogate DABSO is employed in a series of metal-catalyzed transformations to access useful sulfonyl-derived functional groups, such as sulfones, sulfonamides and sulfinate salts. Both palladium and copper catalyzed reactions are described. This work was presented at the 28th International Symposium on the Organic Chemistry of Sulfur, held in Tokyo in the summer of 2018.

\section{GRAPHICAL ABSTRACT}

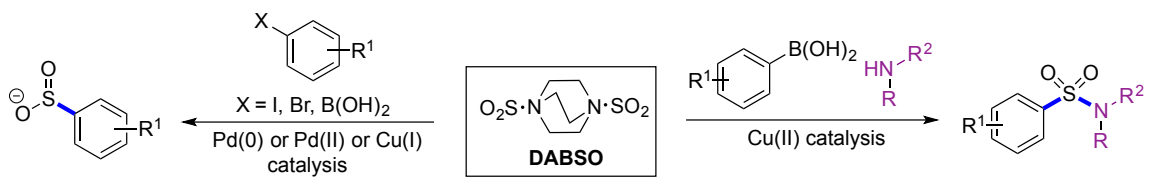

KEYWORDS Palladium catalysis; Copper catalysis; Sulfonamides; Sulfones; Sulfinates

\section{INTRODUCTION}

Sulfonyl-derived functional groups such as sulfones, sulfonamides and sulfonate esters feature in a broad range of bioactive molecules, and are common motifs in pharmaceuticals and agrochemicals (Scheme 1a). ${ }^{1}$ The most common method to introduce a sulfonyl unit into an aromatic molecule is to employ reactions based on electrophilic aromatic substitution processes, ${ }^{2}$ and while these are extremely effective reactions they do have limitations. For example, electronrich aromatics are the preferred substrates and only certain substitution patterns are readily accessible, and these are usually dictated by the nature and position of substituents on the aromatic ring. ${ }^{3}$ These transformations are also associated with strongly acidic reaction conditions.

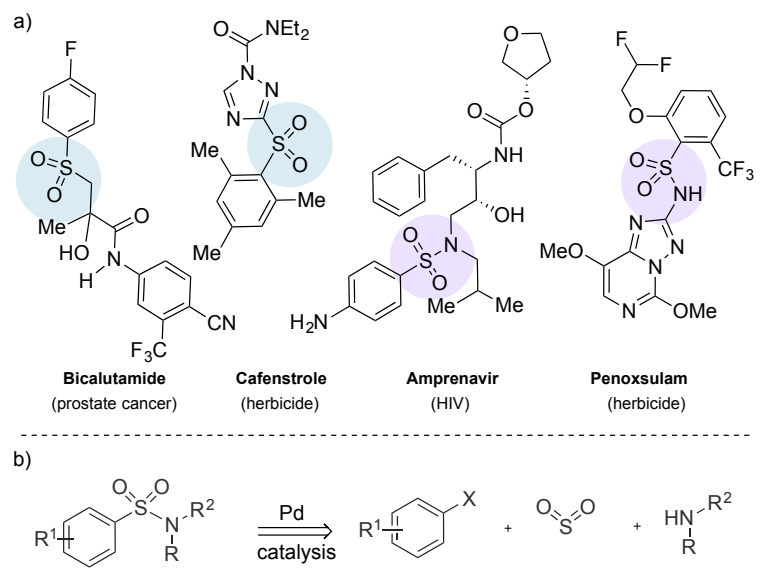

Scheme 1 a) Examples of biologically relevant sulfonyl-containing molecules; b) Our proposed route to sulfonamides.

In order to address several of the limitations associated with classical syntheses of sulfonyl groups, we were interested in developing 
catalytic reactions that allowed the direct

introduction of sulfur dioxide in three-

component reactions. ${ }^{4}$ A proposed disconnection

of a sulfonamide is shown in Scheme 1b. Our

inspiration to explore these reactions comes

from the related chemistry of carbon monoxide,$^{5}$

and also from catalytic reactions that exploit the expulsion of sulfur dioxide in order to achieve

useful bond-forming processes. ${ }^{6}$ There is also a

considerable inorganic literature on the

coordination chemistry of sulfur dioxide. ${ }^{7}$

\section{RESULTS AND DISCUSSION}

We soon established that using sulfur dioxide gas directly in catalytic reactions was challenging, with suppression of all catalytic activity being the most common result. We attributed this to the use of large excesses of the gaseous reagent. In order to avoid using an excess of the reagent, and to simplify the experimental procedures, we began to explore the use of sulfur dioxide surrogates ${ }^{8}$ and were drawn to the use of the Lewis-adduct formed between the diamine $\mathrm{DABCO}$ and two equivalents of sulfur dioxide. ${ }^{9}$ Although this solid, stable adduct has been known since 1988 it had not been used in synthetic chemistry. ${ }^{10}$ While our focus is on the development of new catalytic reactions, we also explored the use of the DABCO-sulfur dioxide adduct, which we have named DABSO, as a sulfur dioxide surrogate for use in non-catalytic reactions.
Scheme 2 gives three examples, all of which proceed by the initial formation of a metal sulfinate (1) from the combination of a preformed organometallic reagent and DABSO. Grignard reagents, organozincs and organolithium reagents can all be employed. The sulfinate intermediates can be readily alkylated to provide sulfones, ${ }^{11}$ combined with amines and sodium hypochlorite to deliver sulfonamides, ${ }^{12}$ or can undergo palladium-catalyzed $S$-arylation to provide diarylsulfones. ${ }^{13} \mathrm{We}$ have also used related chemistry to access sulfoxides. ${ }^{14}$

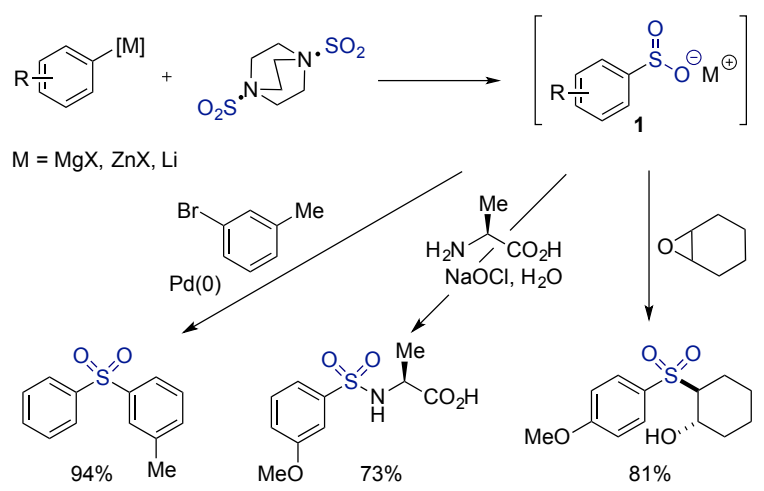

Scheme 2 DABSO used in combination with preformed organometallic reagents.

The first catalytic process we developed allowed the three-component union of aryl halides, sulfur dioxide (from DABSO), and dialkylhydrazines, using a $\operatorname{Pd}(0)$ catalyst, to provide $N$ aminosulfonamides in a single step process (Scheme 4). ${ }^{15}$ These reactions represented the first examples of sulfur dioxide being employed in a cross-coupling type reaction, and served as a proof-of-principle for the processes we were targeting. The limitation of these reactions was 
the need to employ hydrazines as the $N$ nucleophiles, as simple amines were not effective. The Wu group at Fudan University have since reported related reactions, again using hydrazine nucleophiles, but have shown that the inorganic salt $\mathrm{Na}_{2} \mathrm{~S}_{2} \mathrm{O}_{5}$ can function as an effective sulfur dioxide surrogate. ${ }^{16}$ In combination with DABSO, the same group has also reported a related synthesis of $\mathrm{N}$ aminosulfonamides starting from aryl boronic acids. ${ }^{17}$

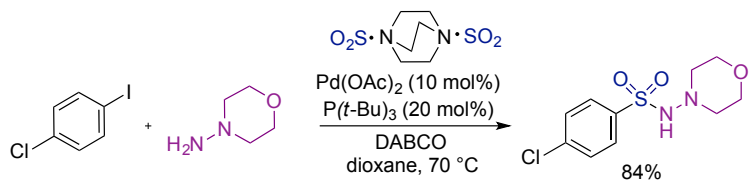

Scheme 3 The palladium-catalyzed synthesis of $N$ aminosulfonamides.

To deliver a more synthetically useful catalytic reaction we next targeted the direct synthesis of sulfinates, starting from aryl halides. After extensive optimization we settled on a reaction system in which isopropanol was used as both the reaction solvent and formal reductant. ${ }^{18}$ The working reaction is shown in Scheme 4, and as can be seen, when the reaction is performed on a gram scale it is possible to use only $1 \mathrm{~mol} \%$ of the palladium catalyst. The desired sulfinates are obtained in good yields but are not the final products isolated. Instead, simple derivatization is used to prepare isolable sulfones and sulfonamides. Chemists at Pfizer in the US have reported a related reaction, which uses $\mathrm{Na}_{2} \mathrm{~S}_{2} \mathrm{O}_{5}$ as the $\mathrm{SO}_{2}$ source and sodium formate as the reductant. ${ }^{19}$

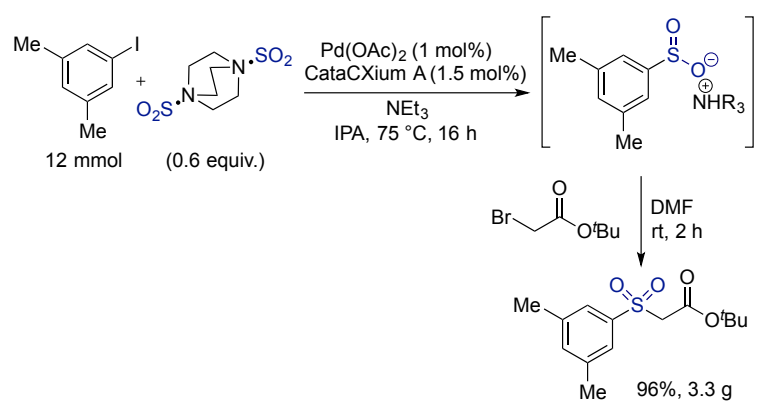

Scheme 4 Palladium(0)-catalyzed sulfinate synthesis from aryl iodides.

Recently, in collaboration with scientists at Pfizer we have adapted our palladium-catalyzed sulfinate synthesis to the preparation of sulfonyl fluorides. ${ }^{20}$ We identified NFSI as a suitable $\mathrm{F}^{+}$ reagent, allowing the smooth conversion of sulfinates into the required sulfonyl fluorides. During reaction optimization we also reexplored ligand selection, and established that the use of AmPhos allowed the routine use of aryl bromide substrates. As is shown in Scheme 5 , we were able to apply this method to the preparation of complex sulfonyl fluorides derived from pharmaceutical fragments. For related synthesis of sulfonyl fluorides see the report from the Ball laboratory. ${ }^{21}$ 


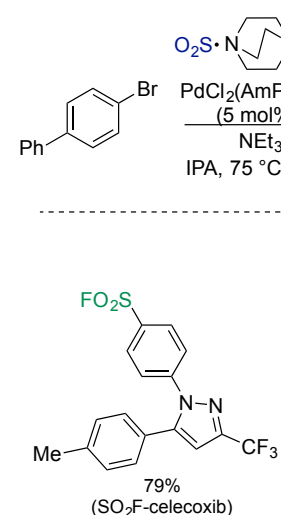

$\mathrm{S} \cdot \mathrm{N} \cdot \mathrm{SO}_{2}$

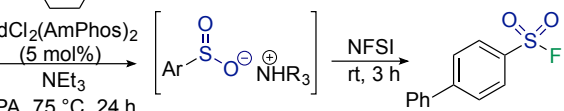

$86 \%$

$6 \%$

$$
\mathrm{Me}_{2} \mathrm{AmPhos}_{\mathrm{NFSI}}^{\mathrm{P}(t-\mathrm{Bu})_{2}}
$$

Scheme 5 Palladium(0)-catalyzed synthesis of sulfonyl fluorides from aryl bromides.

By switching to a palladium(II) catalyst we were able to develop a sulfinate synthesis starting from aryl boronic acids (Scheme 6) ${ }^{22}$ Using the combination of boronic acids and Pd(II) allows a redox-neutral catalytic cycle to operate, in contrast to the $\mathrm{Pd}(0) / \mathrm{Pd}(\mathrm{II})$ cycles involved in our earlier chemistry. The advantages of the Pd(II) system included shorter reaction times and the use of a phosphine-free catalyst. The optimized reaction was tolerant of a broad range of boronic acids, and as before, simple derivatization was used to convert the generated sulfinates into isolable products. Gram scale reactions allowed only $1 \mathrm{~mol} \%$ of palladium catalyst to be used. A related reaction, which employs $\mathrm{Na}_{2} \mathrm{~S}_{2} \mathrm{O}_{5}$ as the $\mathrm{SO}_{2}$ source has been reported. ${ }^{23}$

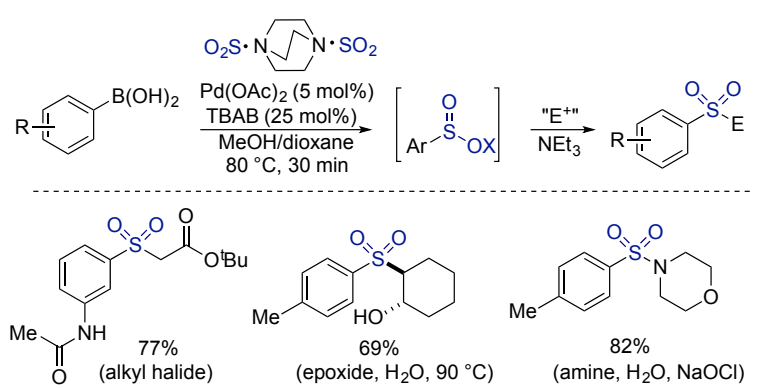

Scheme 6 Palladium(II)-catalyzed sulfinate synthesis from aryl boronic acids.

An attractive feature of using aryl boronic acids as starting materials is that alternative metal catalysts are known to undergo ready transmetallation with these species. The Toste group has used this to develop a gold(I)catalyzed sulfinate synthesis. ${ }^{24} \mathrm{We}$ chose to focus on a more abundant metal, and have developed a copper(I)-catalysed sulfinate synthesis (Scheme 7). ${ }^{25}$ A simple commercially available $\mathrm{Cu}(\mathrm{I})$ salt, used in combination with stoichiometric $\mathrm{Na}(\mathrm{I})$, allowed efficient conversion of boronic acids into sulfinates, and on to sulfones, sulfonamides, and sulfonyl fluorides. Using this copper(I) system we were able to prepare a similar range of products to that which is possible using the palladium-based process. An important difference to note is that the copper reactions require significantly longer reaction times (30 mins vs $12 \mathrm{~h}$ ); however, this must be balanced against the lower cost of the copper catalyst. 


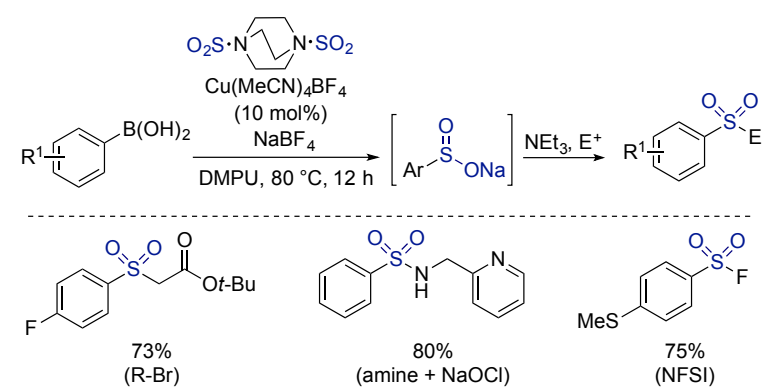

Scheme 7 Copper(I)-catalyzed sulfinate synthesis from aryl boronic acids.

We were able to combine the $\mathrm{Cu}(\mathrm{I})$-catalyzed sulfinate synthesis described above with a $\mathrm{Cu}(\mathrm{I})$ catalyzed sulfinate $S$-arylation reaction, as reported by $\mathrm{Ma},{ }^{26}$ to deliver a sulfonylative variant of the Suzuki-Miyaura cross-coupling (Scheme 8). ${ }^{25}$ The working reaction allows the union of an aryl boronic acid, an aryl iodide and sulfur dioxide (delivered from DABSO). The same commercial $\mathrm{Cu}(\mathrm{I})$ salt was employed as the catalyst, but a supporting bipyridine ligand was needed (Scheme 8). Good variation of both aryl reaction partners was possible. This transformation represents the first example of a sulfonylative version of a classic cross-coupling process. The use of a $\mathrm{Cu}(\mathrm{I})$ catalyst was crucial to the success of these reactions, as attempts to promote similar reactions using palladium catalysts were uniformly unsuccessful.

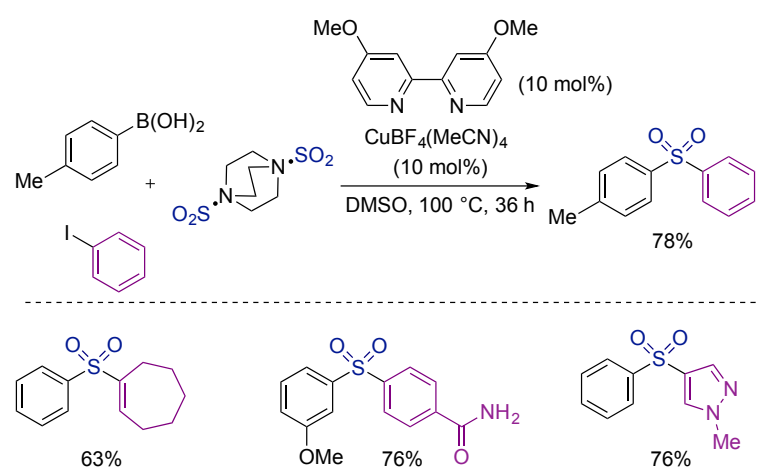

Scheme 8 Copper(I)-catalyzed sulfonylative Suzuki-

Miyaura coupling.

Having established that using copper catalysts delivered distinct reactivity relative to palladium, we continued to explore new reactions using copper. In particular, we have developed a $\mathrm{Cu}(\mathrm{II})$-catalyzed reaction that delivers sulfonamides in a single step from the combination of aryl boronic acids, amines and sulfur dioxide (from DABSO). ${ }^{27}$ The optimized transformation is shown in Scheme 9; $\mathrm{Cu}(\mathrm{OTf})_{2}$ and a bipyridine ligand generate the active catalyst, and the use of DMSO as solvent is crucial. Although we have not undertaken extensive mechanistic studies on the process, our initial experiments are consistent with a ChanLam-type reaction. ${ }^{28}$ Using this reaction we were able to prepare a broad range of sulfonamides, including several that feature medicinally relevant structures. 


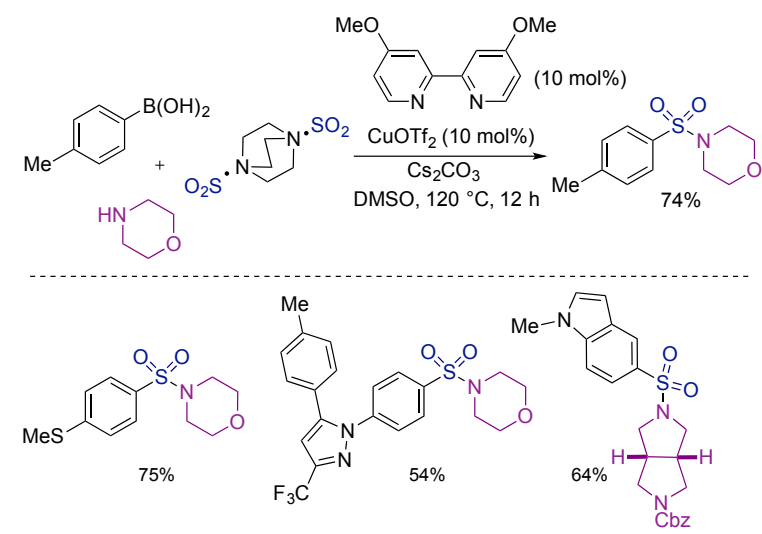

Scheme 9 Copper(II)-catalyzed sulfonamide

synthesis from aryl boronic acids, amines and sulfur dioxide.

\section{CONCLUSIONS}

By employing the solid sulfur dioxide surrogate $\mathrm{DABSO}$, we have been able to develop a series of sulfonylative catalytic processes. Sulfinate salts can be prepared from aryl halides or aryl boronic acids, using palladium or copper catalysts. We have also developed a copper-catalyzed sulfonylative Suzuki-Miyaura cross-coupling reaction, and

(1) a) Scott, K. A.; Njardarson, J. T. Analysis of US FDA-Approved Drugs Containing Sulfur Atoms, Top. Curr. Chem. (Z) 2018, 376; b) Kalgutkar, A. S.; Jones, R.; Sawant, A. In Metabolism, Pharmacokinetics and Toxicity of Functional Groups: Impact of the Building Blocks of Medicinal Chemistry on Admet; Smith, D. A., Ed.; Royal Soc Chemistry: Cambridge, 2010, p 210.

(2) Schmitt, A.-M. D.; Schmitt, D. C. In Synthetic Methods in Drug Discovery: Volume 2; The Royal Society of Chemistry: 2016; Vol. 2, p 123.

(3) Bassin, J. P.; Cremlyn, R. J.; Swinbourne, F. J. Chlorosulfonation of Aromatic and Heteroaromatic Systems, Phosphorus Sulfur Silicon Relat. Elem. 1991, 56, 245.

(4) Deeming, A. S.; Emmett, E. J.; Richards-Taylor, C. S.; Willis, M. C. Rediscovering the Chemistry of Received xx yyyy 2018; accepted xx yyyy 2018, International Conference on Phosphorus Chemistry 2018 Insert acknowledgements and thanks also a copper-catalyzed sulfonamide synthesis based on Chan-Lam reactivity. Taken together these reactions provide access to a broad range of sulfonyl functional groups and should find utility in pharmaceutical and agrochemical research.

\section{ACKNOWLEDGEMENTS}

I would like to thank my current and former co-workers, whose names appear in the references, for their dedication and hard work in delivering the chemistry reported here. I am also very grateful to Professors Kei Goto and Hideki Yorimitsu for their organization of a wonderful ISOCS-28 meeting in Tokyo.

\section{REFERENCES}

Sulfur Dioxide: New Developments in Synthesis and Catalysis, Synthesis 2014, 46, 2701.

(5) Barnard, C. F. J. Palladium-Catalyzed Carbonylation - A Reaction Come of Age, Organometallics 2008, 27, 5402.

(6) Vogel, P.; Turks, M. r.; Bouchez, L.; Marković, D.; Varela-Álvarez, A.; Sordo, J. Á. New Organic Chemistry of Sulfur Dioxide, Acc. Chem. Res. 2007, 40, 931.

(7) Schenk, W. A. Sulfur Oxides as Ligands in Coordination Compounds, Angew. Chem. Int. Ed. 1987, 26, 98.

(8) Emmett, E. J.; Willis, M. C. The Development and Application of Sulfur Dioxide Surrogates in Synthetic Organic Chemistry, Asian. J. Org. Chem. $\mathbf{2 0 1 5}, 4,602$. 
(9) Emmett, E. J.; Willis, M. C. DABCO-bis(sulfur dioxide), DABSO, as an easy-to-handle source of SO2: Sulfonamide preparation, Organic Syntheses 2014, 91, 125.

(10) Santos, P. S.; Mello, M. T. S. The Raman spectra of some molecular complexes of 1azabicyclo[2.2.2] octane and 1,4diazabicyclo[2.2.2] octane, J. Mol. Struct. 1988, 178, 121.

(11) a) Deeming, A. S.; Russell, C. J.; Hennessy, A. J.; Willis, M. C. DABSO-Based, Three-Component, One-Pot Sulfone Synthesis, Org. Lett. 2014, 16, 150; b) Rocke, B. N.; Bahnck, K. B.; Herr, M.; Lavergne, S.; Mascitti, V.; Perreault, C.; Polivkova, J.; Shavnya, A. Synthesis of Sulfones from Organozinc Reagents, DABSO, and Alkyl Halides, Org. Lett. 2014, 16, 154. (12) Deeming, A. S.; Russell, C. J.; Willis, M. C. Combining Organometallic Reagents, the Sulfur Dioxide Surrogate DABSO, and Amines: A One-Pot Preparation of Sulfonamides, Amenable to Array Synthesis, Angew. Chem. Int. Ed. 2015, 54, 1168. (13) Emmett, E. J.; Hayter, B. R.; Willis, M. C. Palladium-Catalyzed Three-Component Diaryl Sulfone Synthesis Exploiting the Sulfur Dioxide Surrogate DABSO, Angew. Chem. Int. Ed. 2013, 52, 12679.

(14) Lenstra, D. C.; Vedovato, V.; Ferrer Flegeau, E.; Maydom, J.; Willis, M. C. One-Pot Sulfoxide Synthesis Exploiting a Sulfinyl-Dication Equivalent Generated from a DABSO/Trimethylsilyl Chloride Sequence, Org. Lett. 2016, 18, 2086.

(15) Nguyen, B.; Emmett, E. J.; Willis, M. C. Palladium-Catalyzed Aminosulfonylation of Aryl Halides, J. Am. Chem. Soc. 2010, 132, 16372. (16) Ye, S.; Wu, J. A palladium-catalyzed reaction of aryl halides, potassium metabisulfite, and hydrazines, Chem. Commun. 2012, 48, 10037. (17) Ye, S.; Wu, J. A palladium-catalyzed threecomponent coupling of arylboronic acids, sulfur dioxide and hydrazines, Chem. Commun. 2012, 48, 7753.

(18) Emmett, E. J.; Hayter, B. R.; Willis, M. C. Palladium-Catalyzed Synthesis of Ammonium Sulfinates from Aryl Halides and a Sulfur Dioxide
Surrogate: A Gas- and Reductant-Free Process, Angew. Chem. Int. Ed. 2014, 53, 10204.

(19) Shavnya, A.; Coffey, S. B.; Smith, A. C.; Mascitti, V. Palladium-Catalyzed Sulfination of Aryl and Heteroaryl Halides: Direct Access to Sulfones and Sulfonamides, Org. Lett. 2013, 15, 6226.

(20) Davies, A. T.; Curto, J. M.; Bagley, S. W.; Willis, M. C. One-pot palladium-catalyzed synthesis of sulfonyl fluorides from aryl bromides, Chem. Sci. 2016, 8, 1233.

(21) Tribby, A. L.; Rodriguez, I.; Shariffudin, S.; Ball, N. D. Pd-Catalyzed Conversion of Aryl Iodides to Sulfonyl Fluorides Using $\mathrm{SO}_{2}$ Surrogate DABSO and Selectfluor, J. Org. Chem. 2017, 82, 2294.

(22) Deeming, A. S.; Russell, C. J.; Willis, M. C. Palladium(II)-Catalyzed Synthesis of Sulfinates from Boronic Acids and DABSO: A Redox-Neutral, Phosphine-Free Transformation, Angew. Chem. Int. Ed. 2016, 55, 747.

(23) Shavnya, A.; Hesp, K. D.; Mascitti, V.; Smith, A. C. Palladium-Catalyzed Synthesis of (Hetero)Aryl Alkyl Sulfones from (Hetero)Aryl Boronic Acids, Unactivated Alkyl Halides, and Potassium Metabisulfite, Angew. Chem. Int. Ed. 2015, 54, 13571.

(24) Johnson, M. W.; Bagley, S. W.; Mankad, N. P.; Bergman, R. G.; Mascitti, V.; Toste, F. D. Application of fundamental organometallic chemistry to the development of a gold-catalyzed synthesis of sulfinate derivatives, Angew. Chem. Int. Ed. 2014, $53,4404$.

(25) Chen, Y.; Willis, M. C. Copper(I)-catalyzed sulfonylative Suzuki-Miyaura cross-coupling, Chem. Sci. 2017, 8, 3249.

(26) Zhu, W.; Ma, D. Synthesis of Aryl Sulfones via l-Proline-Promoted CuI-Catalyzed Coupling Reaction of Aryl Halides with Sulfinic Acid Salts, J. Org. Chem. 2005, 70, 2696.

(27) Chen, Y.; Murray, P. R. D.; Davies, A. T.; Willis, M. C. Direct Copper-Catalyzed ThreeComponent Synthesis of Sulfonamides, J. Am. Chem. Soc. 2018, 140, 8781.

(28) Lam, P. Y. S. In Synthetic Methods in Drug Discovery: Volume 1; The Royal Society of Chemistry: 2016; Vol. 1, p 242. 